\title{
BMJ Open Factors shaping the implementation and use of Clinical Cancer Decision Tools by GPs in primary care: a qualitative framework synthesis
}

\author{
Paula Theresa Bradley (D , ${ }^{1}$ Nicola Hall, ${ }^{2}$ Gregory Maniatopoulos, ${ }^{3}$ \\ Richard D Neal (D) , ${ }^{4}$ Vinidh Paleri, ${ }^{5}$ Scott Wilkes ${ }^{1}$
}

To cite: Bradley PT, Hall N, Maniatopoulos G, et al. Factors shaping the implementation and use of Clinical Cancer Decision Tools by GPs in primary care: a qualitative framework synthesis. BMJ Open 2021;11:e043338. doi:10.1136/ bmjopen-2020-043338

- Prepublication history and additional material for this paper are available online. To view these files, please visit the journal online (http://dx.doi. org/10.1136/bmjopen-2020043338).

Received 04 August 2020 Revised 12 January 2021 Accepted 30 January 2021

Check for updates

C Author(s) (or their employer(s)) 2021. Re-use permitted under CC BY-NC. No commercial re-use. See rights and permissions. Published by BMJ.

${ }^{1}$ Medical School, University of Sunderland, Sunderland, UK

${ }^{2}$ Faculty of Medical Sciences, University of Newcastle upon Tyne, Newcastle upon Tyne, UK ${ }^{3}$ Newcastle Business School, Northumbria University, Newcastle upon Tyne, UK ${ }^{4}$ Institute of Health Sciences, University of Leeds, Leeds, UK

${ }^{5}$ Head and Neck Unit, Royal Marsden Hospital NHS Trust, London, UK

Correspondence to Dr Paula Theresa Bradley; paula_bradley@me.com

\section{ABSTRACT}

Objective Clinical Cancer Decision Tools (CCDTs) aim to alert general practitioners (GPs) to signs and symptoms of cancer, supporting prompt investigation and onward referral. CCDTs are available in primary care in the UK but are not widely utilised. Qualitative research has highlighted the complexities and mechanisms surrounding their implementation and use; this has focused on specific cancer types, formats, systems or settings. This study aims to synthesise qualitative data of GPs' attitudes to and experience with a range of CCDTs to gain better understanding of the factors shaping their implementation and use.

Design A systematic search of the published (MEDLINE, CINAHL, Web of Science and EMBASE) and grey literature (July 2020). Following screening, selection and assessment of suitability, the data were analysed and synthesised using normalisation process theory.

Results Six studies (2011 to 2019), exploring the views of GPs were included for analysis. Studies focused on the use of several different types of CCDTs (Risk Assessment Tools (RAT) or electronic version of RAT (eRAT), QCancer and the 7-point checklist). GPs agreed CCDTs were useful to increase awareness of signs and symptoms of undiagnosed cancer. They had concerns about the impact on trust in their own clinical acumen, whether secondary care clinicians would consider referrals generated by CCDT as valid and whether integration of the CCDTs within existing systems was achievable.

Conclusions CCDTs might be a helpful adjunct to clinical work in primary care, but without careful development to legitimise their use GPs are likely to give precedence to clinical acumen and gut instinct. Stakeholder consultation with secondary care clinicians and consideration of how the CCDTs fit into a GP consultation are crucial to successful uptake. The role and responsibilities of a GP as a clinician, gatekeeper, health promoter and resource manager affect the interaction with and implementation of innovations such as CCDTs.

\section{INTRODUCTION}

Cancer survival in the UK is below that of European counterparts. ${ }^{1}$ One factor leading to poor outcome is the stage of cancer at diagnosis, a later stage at diagnosis makes optimum treatment more difficult. An ambition of The National Health Service (NHS) Long-Term Plan is to diagnose $75 \%$ of cancers at stage 1 or 2 by $2028 .{ }^{2}$ Improvements in rates of smoking and obesity aim to reduce the lifetime risk of developing cancer and, improving uptake of screening aims to diagnose presymptomatic cancers. Improving early cancer diagnosis is one of the quality improvement tenets of the NHS England general practitioner (GP) Contract 2020/2021 and suggested areas for review include referral practice and use of Clinical Cancer Decision Tools (CCDTs). ${ }^{3}$

CCDTs are intended to alert GPs to suspicious early signs and symptoms of an undiagnosed cancer. A number of CCDTs have been developed for use in primary care in England over the past 15 years to help GPs consider decisions about onward referral for investigation or specialist assessment. QCancer is embedded in the Egton Medical Information System (EMIS) web electronic patient record system used by GPs in England as well as being available online (www.qcancer.org). A Risk Assessment Tool (RAT), based on case control cohort studies, presented in a paper or mouse-mat form were sent to English GP practices in 2012. The two (QCancer and eRAT) were incorporated into GP software systems in 2013 and called 'electronic cancer decision support tools for cancer'. These support tools were evaluated as part of a Cancer Research UK and MacMillan study. ${ }^{4}$ The 7-point checklist (7PCL) is a validated diagnostic aid integrated into EMIS for assessment of pigmented skin lesions. ${ }^{56}$

Despite efforts to assess and establish evidence for the use of CCDTs, findings from a UK-wide survey of GPs on CCDTs ${ }^{78}$ suggest that, of those practices with access to an electronic version of CCDTs, only a third have downloaded or activated one through their 
clinical record systems. The survey responses suggest that 'levels of tool uptake are relatively low'. 'A recent systematic review ${ }^{9}$ of CCDTs to investigate whether they improve diagnostic decision making for cancer in primary care demonstrates their potential but note that effective implementation is still elusive.

Drawing on normalisation process theory (NPT), this study explores factors shaping the implementation and use of CCDTs in primary care. NPT is a framework designed to contextualise, understand and evaluate "the processes (implementation) by which new health technologies and other complex interventions are routinely operationalised in everyday work (embedding) and sustained in practice (integration).${ }^{10}$ NPT provides a set of conceptual tools to aid understanding of this dynamic process. It considers four theoretical constructs: (1) coherence, (2) cognitive participation, (3) collective action and (4) reflexive monitoring (4-6).

\section{Aim}

This study aims to synthesise qualitative research on GPs' attitudes to, and experiences of CCDTs. Drawing on a framework synthesis approach, it aims to provide a comprehensive analysis of factors shaping the implementation and use of CCDTs informed by NPT (6).

\section{METHODS}

\section{Data sources and search strategy}

A systematic electronic literature search from inception to July 2020 in MEDLINE, CINAHL, Web of Science and EMBASE databases was undertaken. A comprehensive predefined search strategy was developed by PTB using the SPICE (Setting Population Intervention Comparison Evaluation ${ }^{11}$ box 1) approach. No publication type, language or date limits were applied to the searches. PTB carried out online searches via Google for grey literature using combinations of the terms from the electronic

\section{Box 1 SPICE criteria}

\section{Setting}

General practice/primary care in National Health Service /Western type public health carehealthcare system (primary care gatekeeper)

Population.

General practitioners/primary care doctors/family doctors.

\section{Intervention}

Cancer Clinical Decision Tools-paper, electronic, desktop, mousemap, electronic decision aid/system.

\section{Comparison}

Normal practice/no comparator.

Other form of risk assessment.

\section{Evaluation}

Qualitative-face-to-face interviews, telephone interviews, questionnaires, focus groups, direct observation.

SPICE, Setting Population Intervention Comparison Evaluation. database searches. Email contact was made with two authors to establish that multiple publications were from the same study and another author was contacted to obtain a copy of a PhD thesis. Manual searches were undertaken by reviewing the reference lists of relevant identified literature from the database search results.

An example of the search strategy for EMBASE can be found in online supplemental material.

\section{Selection criteria}

Studies were included if they reported qualitative analysis (including qualitative aspects of mixed-methods studies) of GP's participation and engagement with CCDTs. Studies which used face-to-face or telephone interviews, questionnaires, focus groups or direct observation were eligible. CCDTs were defined as any tool (digital, paper, electronic) used within a consultation which provided an outcome measure such as a percentage risk or a recommendation to the doctor to consider an underlying cancer diagnosis as the cause for a patient's signs or symptoms.

PTB and NH independently screened titles, abstracts and full articles using the inclusion criteria. Any differences in selections of full texts for inclusion were discussed until agreement on inclusion or exclusion was reached (full-text exclusions with reasons is available on request). An independent reviewer was available, this was not required.

\section{Data extraction}

For each article, all text from 'Results/Findings' and 'Discussion' were extracted and imported into NVivo V.11 software (NVivo Qualitative data analysis Software; QSR International, V.11, 2016). Study characteristics were extracted into a spreadsheet to explore potential associations between specific themes and studies.

\section{Critical appraisal}

All included studies were critically appraised by РТВ and NH using the Critical Appraisal Skills Programme checklist (CASP) for qualitative research. ${ }^{12}$ Any discrepancies were resolved through discussion, until consensus was reached. The studies were not excluded or weighted based on the quality of reporting or any hierarchy of qualitative evidence. $^{13}$

\section{Data synthesis}

Following familiarisation with the selected studies, PTB and $\mathrm{NH}$ developed a thematic framework to apply to the studies. The data were coded and charted line by line by PTB and NH. Themes and interpretation were discussed and refined with GM and mapped onto the NPT framework. ${ }^{14}$ This work was aided by use of the adapted domain questions in table 1 . The final analysis was discussed and validated by all authors.

\section{Patient and public involvement}

Patients and the public were not involved in the review. 
Table 1 Domains of NPT in relation to CCDT

\begin{tabular}{|c|c|}
\hline NPT domains & Questions \\
\hline $\begin{array}{l}\text { Coherence } \\
\text { (Meaning and sense making } \\
\text { of participants) } \\
\text { How they make sense of } \\
\text { the work of implementation } \\
\text { and integration in order } \\
\text { to promote/inhibit routine } \\
\text { embedding of a practice. }\end{array}$ & $\begin{array}{l}\text { Is the CCDT easy to describe? } \\
\text { Do GPs understand what the CCDT is? } \\
\text { Do GPs understand how the CCDT should be implemented? } \\
\text { Is the CCDT clearly distinct from other practices? } \\
\text { Do GPs express understanding of how the CCDT is distinct from other practices? } \\
\text { Does the CCDT have a clear purpose for GPs/patients? } \\
\text { Do GPs report a shared understanding of the purpose/benefit/value of the CCDT? } \\
\text { What benefits do GPS feel the CCDT will bring and to whom? (GPs/patients)? } \\
\text { Are these benefits valued by GPs? } \\
\text { Does the CCDT fit with the overall goals and activity of the organisation (practice/NHS) } \\
\text { Do GPs feel the CCDT fits with their own responsibilities/ roles? }\end{array}$ \\
\hline $\begin{array}{l}\text { Cognitive participation } \\
\text { (Commitment and } \\
\text { engagement by participants) } \\
\text { Process and work go } \\
\text { through to enrol individuals } \\
\text { to engage with new practice }\end{array}$ & $\begin{array}{l}\text { Do GPs think the CCDT a good idea-'buy in'? } \\
\text { Do GPs see the point of the CCDT easily? } \\
\text { Are GPs willing to drive implementation? } \\
\text { Are GPs able to/willing to sustain involvement? } \\
\text { Do GPs feel is it 'right'/legitimate they are involved? } \\
\text { Do GPs feel using CCDTs is a legitimate part of their role? }\end{array}$ \\
\hline $\begin{array}{l}\text { Collective action } \\
\text { (The work participants } \\
\text { (individuals and } \\
\text { organisations) do to make } \\
\text { the intervention function) } \\
\text { How they enact it }\end{array}$ & $\begin{array}{l}\text { What effect does the CCDT have on the work of GPs (how the CCDT affects the consultation)? } \\
\text { Does the CCDT promote or impede GPs work? } \\
\text { How compatible is the CCDT with existing work practices? } \\
\text { Does it make work easier? } \\
\text { How does it affect their roles/responsibilities/training needs? } \\
\text { Do GPs require extensive training before they can use the CCDT? } \\
\text { Is there organisational support for the CCDT? } \\
\text { Is there confidence in the new practice when they are using/enacting it? } \\
\text { What impact does the CCDT have on division of labour, resources, power and responsibility } \\
\text { between professional groups? } \\
\text { Is there confidence in the new practice when they are using/enacting it? }\end{array}$ \\
\hline
\end{tabular}

CCDT, Clinical Cancer Decision Tool; GPs, general practitioners; NHS, National Health Service; NPT, normalisation process theory.

\section{RESULTS}

The Preferred Reporting Items for Systematic Reviews and Meta-Analyses diagram (figure 1) shows the results of the searches. Five published peer-reviewed articles, two reports and one $\mathrm{PhD}$ thesis were eligible for analysis. ${ }^{415-20}$ Two of the published papers came from the same study these have been considered as one dataset. ${ }^{1819}$ Another of the published articles and one of the reports were based on the same study data. ${ }^{16} 17$ The studies were published between 2011 and 2019.

\section{Characteristics of included studies}

The studies were heterogeneous in design, CCDT used and analysis. One study was from Australia ${ }^{8}$ and the others were from the UK. ${ }^{415-20}$ Data from 107 GPs is included in the selected studies (table 2).
The characteristics of the CCDTs assessed in the studies are summarised in table 3 . These included RAT/eRAT, ${ }^{1819}$ QCancer ${ }^{8}$ two studies used both of these ${ }^{415}$ and one used the $7 \mathrm{PCL}^{20}$ (table 4). RAT/eRAT are CCDTs based on the results of population-based case-control studies, QCancer is based on statistically validated cohort studies, and the 7PCL is a check box CCDT intended to be used as a support to GP decision making about referral for melanoma. ${ }^{20}$ 7PCL is part of National Institute for Health and Care Excellence (NICE) recommendations for the assessment of pigmented lesions in Primary Care. ${ }^{21}$ The CCDTs were presented to the clinician in different formats; including mouse mats, checklists and integrated computer prompts. 


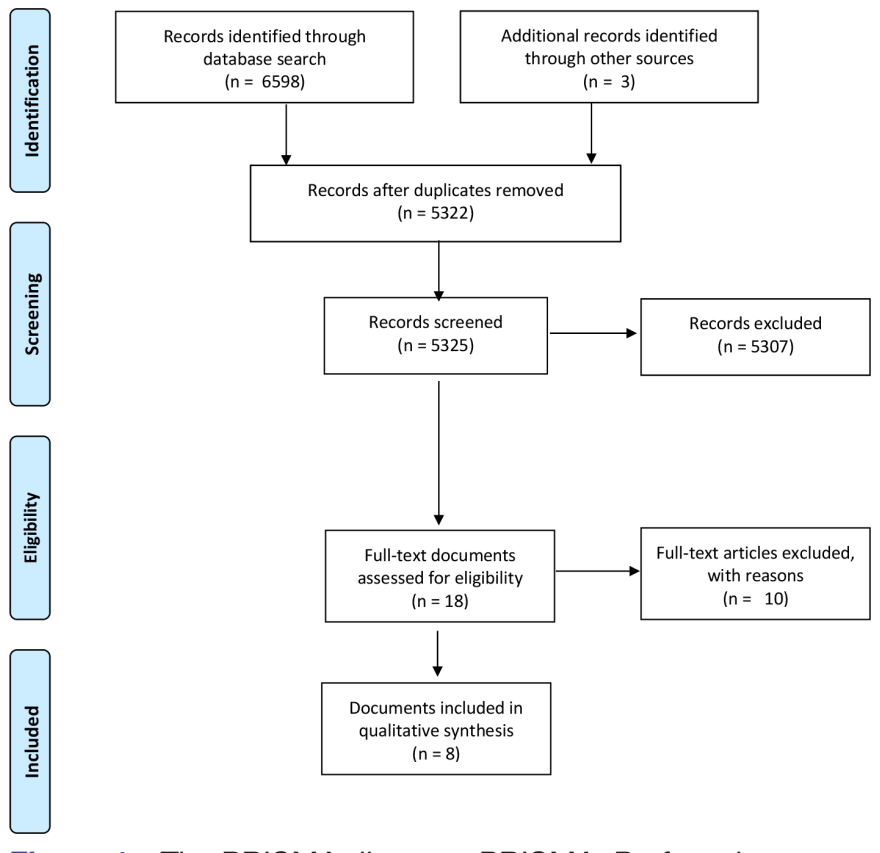

Figure 1 The PRISMA diagram. PRISMA, Preferred Reporting Items for Systematic Reviews and Meta-Analyses.

One study asked GPs to participate in actor simulated cases using QCancer, followed by an interview exploring their experience. ${ }^{8}$ The other studies interviewed GPs after using the CCDTs in normal practice for a period of time. Two of these collected data via semistructured telephone interviews, ${ }^{41617}$ one undertook face-to-face interviews, ${ }^{8}$ another convened a focus group ${ }^{15}$ and one gathered data via a mix of face-to-face and telephone interviews. ${ }^{20}$ The interviews with GPs were conducted at variable time intervals after using the CCDTs ranged from immediately to months later.

All studies used the framework method to analyse data. Underpinning theoretical frameworks included The normalisation process model (an earlier version of NPT), ${ }^{16} 17$ the Consolidated Framework for Implementation Research and Risk Communication Framework. ${ }^{15} 20$ The remaining studies developed their own inductive coding framework. ${ }^{481819}$

In the Australian study, ${ }^{8}$ there was a US\$300 incentive for participants. Practices were given some financial support via the Cancer Networks for participation in another study. ${ }^{18} 19$ These financial reward for participation were neither discussed in terms of its impact on the participants' engagement nor their responses to interview questions.

\section{Quality assessment}

Independent CASP assessment of all included studies, by PTB and NH demonstrated they were of high quality (available as online supplemental material). One omission from all studies was an exploration of the role of the interviewer in relation to the interviewee and any impact this could have on the data (reflexivity). ${ }^{22}$ This failure reflects on potential bias of the study and its conclusions. ${ }^{12}$

\section{Synthesis}

The synthesis focuses on; the CCDT, the role of the doctor, the consultation, the impact on cancer investigation and referrals, and the implications on and influence of secondary care cancer services.

Selected quotes from studies add richness to the framework synthesis. They illustrate the themes and how they map onto the NPT domains. Where available demographic information about the source of the quote and the CCDT that the participant discussed is provided (table 4).

\section{Coherence: the impact of CCDT on the role of the GP}

Coherence refers to how participants make sense of an intervention. ${ }^{18}$ The data suggest that how GPs understand and make sense of CCDTs is inextricably linked to the perceptions of their role as a GP and how this is impacted by their use.

\section{Communicating, sharing and understanding risk}

A key result was that GPs were expected to communicate the pros and cons of a course of action, investigation, treatment and the likelihood of signs and/or symptoms present because of an undiagnosed cancer. This was influenced by the potential reaction from patients. Different levels of comfort were experienced when using CCDTs within consultations ${ }^{815}$ because of the perceived impact of the information on the patient, how it was communicated and what reaction that might elicit.

Some GPs had concerns that using the CCDT would increase patient anxiety so did not share the cancer risk figures, even keeping risk figures hidden from the patient's view, or feeling that using the risk percentages in discussion with the patient would be challenging. ${ }^{4}$ Others were comfortable using the CCDT to reassure patients. ${ }^{1520}$

There was a lack of understanding about the intended role of the CCDT or how to use the results that it generated. ${ }^{4}$ 15-17 This misunderstanding may reflect poor knowledge of how to interpret the results generated from a CCDT. Understanding how the CCDT fits within the current cancer pathway, how the cancer risk figures were calculated, might improve confidence in the validity of using a CCDT. ${ }^{8-17}$ Integration into the consultation might be encouraged by such knowledge.

\section{Collaboration and involvement with secondary care and existing guidelines}

A common theme across studies was the reported need for the CCDT to be integrated within existing referral pathways and endorsed by secondary care. ${ }^{4}{ }^{15-17}$ GPs worried that a change in referral patterns, as a result of its use, might not be well received by hospital specialty team with no knowledge of it. Some participants felt uneasy using the CCDT to make referral decisions as a consequence. Having secondary care doctors' endorsement of the CCDT appears to be an important aspect in promoting its routine use for GPs. Justification of referrals 


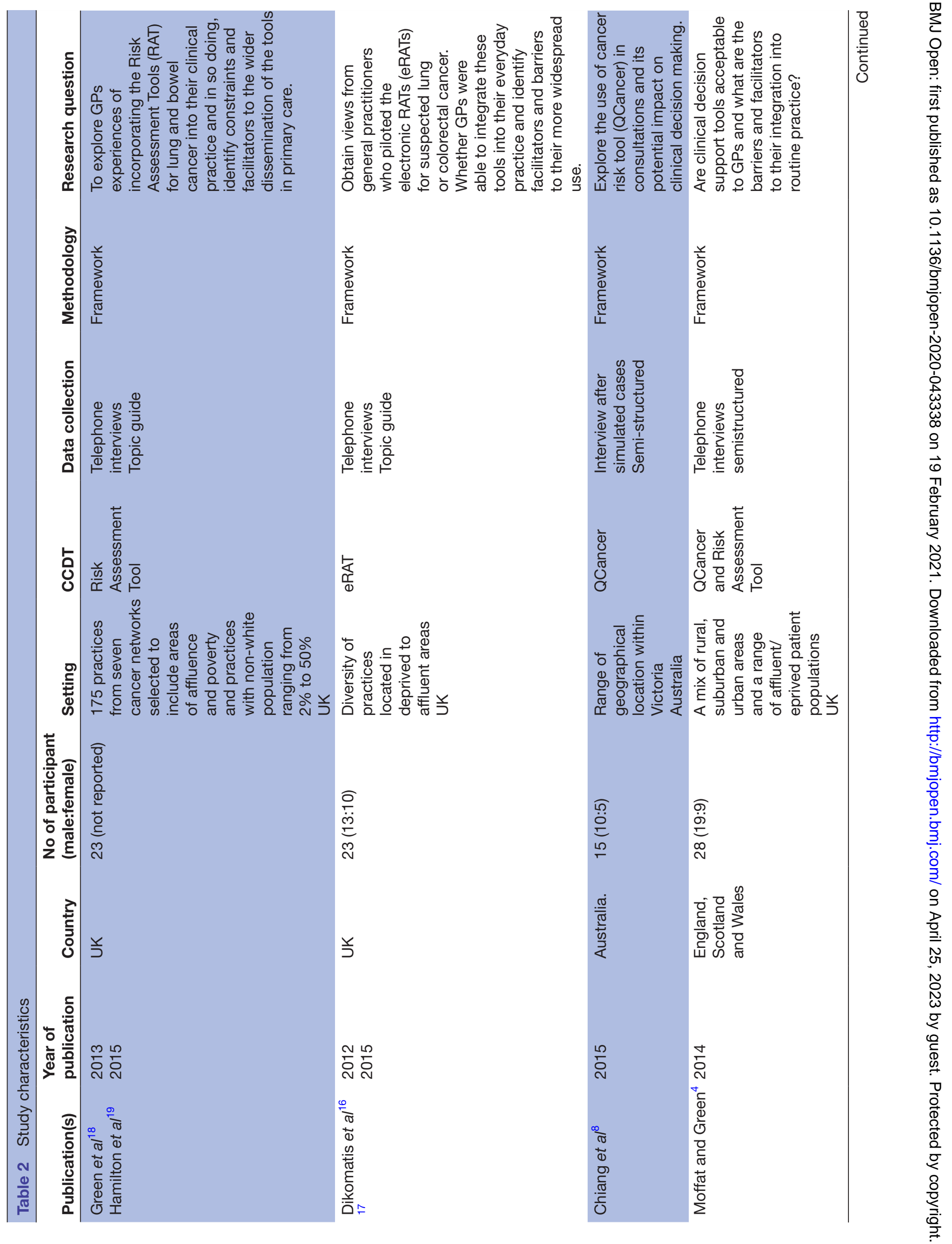




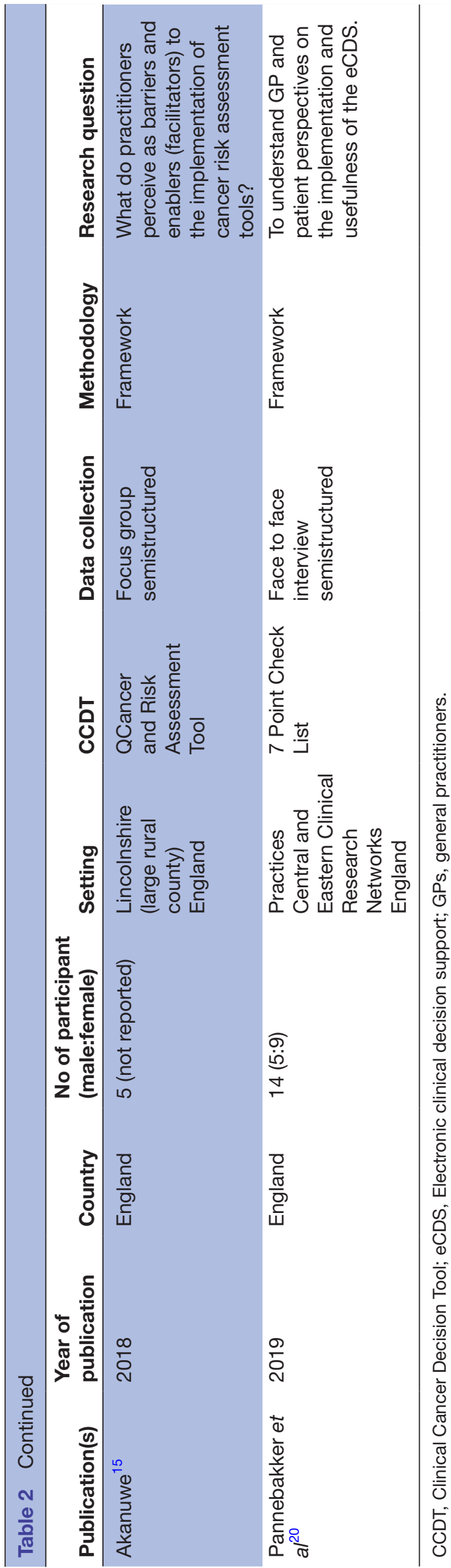

for suspected cancer using the percentages generated by the CCDT would require some knowledge of the CCDT by secondary care. ${ }^{415-17}$

Participants in one study questioned the legitimacy of integration of a checklist in the clinical system which already existed within local pathways and on referral forms. ${ }^{16}$ Participants commented that using a CCDT to determine referral decisions potentially threatens the professional identity and clinical reputation of a GP possibly undermining their relationship to secondary care.

The 'one size fits all' approach to training

Use of the CCDT by GPs was determined by their involvement in a research study, ${ }^{15-17}$ the training they had prior to using the CCDT and the support that they received while using the CCDT.

Training is essential in determining coherence. ${ }^{4}$ It affects how GPs understand the CCDT, what makes the CCDT distinct from other practices and how it may benefit both the doctor and patient. Any training should aim to demonstrate how the CCDT fits with the responsibilities and role of the doctor as well as the overall goals of the NHS in terms of timely diagnosis of cancer.

\section{Cognitive participation: elements determining GPs use of CCDT}

Cognitive participation refers to the commitment and engagement with an intervention. ${ }^{18}$ Two influences on engagement with CCDTs were identified.

\section{Clinical acumen vs protocol}

Some participants commented on their discomfort using the CCDT to aid their decision making rather than it being an instruction that had to be obeyed. Other GPs made it clear that they did not want to have their clinical acumen challenged by a CCDT. ${ }^{415-171920}$ Protocolisation was one of the most commonly discussed factors affecting implementation. ${ }^{4815-171920}$ GP responses suggest a reluctance to be protocol driven in their decision making. This questions the perceived legitimacy of the use of CCDTs when GPs do not feel it 'right' that they should be used. ${ }^{18}{ }^{19}$ Some GPs did overrule the outcomes generated by the CCDT if these were not consistent with their clinical impression. ${ }^{4815} 20$

The CCDT was used to back up 'instinct' and ignored if it did not. ${ }^{40}$ Despite this theme being a dominant one, there was little exploration in the studies of whether the CCDT was used by GPs to justify not taking further action in those with a low risk of symptoms being caused by cancer and only one interviewee was quoted alluding to this. ${ }^{8}$ This could be because of assumptions about the underlying research agenda of early recognition and diagnosis rather than an appreciation that CCDTs might actually contribute to a reduction in over-investigation and improved selection of patients for 2-week wait referral pathways. ${ }^{8}$ 
Table 3 CCDTs used in studies

\begin{tabular}{|c|c|c|c|}
\hline Name & Cancer type & Format & Use and development \\
\hline $\begin{array}{l}\text { Risk Assessment Tool } \\
\text { (RAT) }\end{array}$ & Lung, colorectal & Desk based & $\begin{array}{l}\text { Quantifies risk of cancer in symptomatic } \\
\text { primary care patients. } \\
\text { Consists of risk score for high-risk } \\
\text { symptoms in isolation, for repeat } \\
\text { presentation of the same symptom and in } \\
\text { combination with one other symptom. } \\
\text { Positive predictive Values for symptoms } \\
\text { of cancer, developed through series of } \\
\text { population-based case and control studies } \\
\text { in primary care setting. }\end{array}$ \\
\hline Electronic RAT & $\begin{array}{l}\text { Lung (non-smokers), lung } \\
\text { (smokers), colorectal }\end{array}$ & Electronic & $\begin{array}{l}\text { Electronic version of clinical decision Risk } \\
\text { Assessment Tools described above. }\end{array}$ \\
\hline QCancer & $\begin{array}{l}\text { Lung, colorectal, gastro- } \\
\text { oesophageal, pancreatic, blood, } \\
\text { renal, prostate and various others }\end{array}$ & Electronic & $\begin{array}{l}\text { QCancer algorithms can be used to } \\
\text { calculate the percentage probability of } \\
\text { having an undiagnosed cancer. } \\
\text { Developed using QResearch database in } \\
\text { a series of prospective cohort studies, it } \\
\text { incorporates a range of risk factors. }\end{array}$ \\
\hline $\begin{array}{l}\text { Electronic clinical decision } \\
\text { support } 7 \text { Point Check List }\end{array}$ & Melanoma & Electronic & $\begin{array}{l}\text { Electronic Clinical Decision Support for } \\
\text { assessment of pigmented lesions. } \\
\text { Integrated into EMIS clinical system. } \\
\text { A validated diagnostic checklist of } 7 \\
\text { weighted features of a pigmented lesion. } \\
\text { A score of } 3 \text { or more is suspicious of a } \\
\text { diagnosis of melanoma. }\end{array}$ \\
\hline
\end{tabular}

CCDT, Clinical Cancer Decision Tool.

\section{The medicolegal implications of using a new CCDT}

Medicolegal implications of using a CCDT to determine a referral were highlighted by two of the studies. ${ }^{16} 1720$ Uptake could be impeded if GPs felt there was a medicolegal threat to their decision from patients who were later diagnosed with cancer after the CCDT had highlighted an increased risk, but a decision had been made not to investigate or refer.

\section{Collective action: impact of the CCDT on the work of the GP}

Collective action is the work individuals and organisations do to make an intervention work. ${ }^{23}$ The implementation and use of CCDTs within primary care can impact on the work of a GP in a number of ways.

\section{Increasing awareness}

GPs found the use of CCDTs in clinical practice as beneficial. Using them increased their awareness of cancer symptoms. For some GPs, the CCDT acted as a reminder of suspicious signs and symptoms. ${ }^{4815-19}$ This was considered important for trainees and GPs less experienced in dermatology in the case of the 7PCL. ${ }^{20}$ There was appreciation that some patients may not otherwise have had such a prompt referral to cancer services. The use of the CCDT increased awareness, thus building confidence in NICE guidelines. ${ }^{416}$

\section{Prompt fatigue}

Prompt fatigue because of CCDT generated reminders was mentioned by several studies. ${ }^{416} 17$ The electronic interruptions impacted on the flow of the consultation. The prompts were regarded, in some studies, as a nuisance making work more difficult ${ }^{1617}$ another commented on the usefulness of prompts for future consultations. ${ }^{20}$

\section{Impact of IT integration}

A major component of how easy it was for GPs to engage with the CCDT was software integration with existing clinical systems. GPs commented on clunky working and lack of support to operate the CCDT within the existing IT system. ${ }^{4-17}$ One study highlighted that the explosion of decision aids in clinical systems means they are sometimes available, but the clinician is unaware of their existence. ${ }^{20}$

Integration into clinical IT system was an issue which had the potential to 'make or break' implementation. When participants encountered problems with using the CCDT it was sometimes met with frustration, some GPs chose to abandon use. ${ }^{4}$ Time as a resource

Time and capacity issues was one of the most commonly discussed factors shaping implementation ${ }^{4{ }^{15-20}}$ and a frequent response to questions involving any assessment of healthcare technology implementation. Recognition of the benefits of using a CCDT was essential to justify the additional time required for its use. This impacts on 
Table 4 Table of quotes to illustrate the themes

Domain Theme Quote Source

Coherence

Communicating, sharing 'Sometimes I hide it, just in case I cause an alarm, but Male GP eRAT' and understanding risk I will start to cover it during the consultation if there is any risk, yes. It depends because, you know, some patients, if they're anxious, when they see something like that, they become more anxious'

\begin{tabular}{|c|c|c|}
\hline & $\begin{array}{l}\text { 'If someone was very worried and they scored zero } \\
\text { then I might be able to say, 'Look, this is a scoring } \\
\text { system that's been developed,' and it might just aid } \\
\text { reassurance. Equally, if I was worried...I might just say, } \\
\text { 'Look, this is the scoring system, you've got quite a lot } \\
\text { of points on this. It doesn't mean it's anything serious } \\
\text { but it does mean we need to look into it more closely" }\end{array}$ & $\begin{array}{l}\text { Male GP } 40 \text { years old } \\
7 \mathrm{PCL}^{20}\end{array}$ \\
\hline \multirow[t]{2}{*}{$\begin{array}{l}\text { Collaboration and } \\
\text { involvement with } \\
\text { secondary care and } \\
\text { existing guidelines }\end{array}$} & $\begin{array}{l}\text { 'My concern is that the tools are not known to the } \\
\text { secondary or hospital setup. So, I referred some } \\
\text { patients, and I am concerned they may not recognise } \\
\text { my QCancer referral...So, when I am thinking, if they } \\
\text { see the patients I referred using QCancer, they will } \\
\text { ask-who is this? Is this a new doctor, a new GP?' }\end{array}$ & GP, QCancer ${ }^{15}$ \\
\hline & $\begin{array}{l}\text { 'There are criterion boxes often and very occasionally } \\
\text { a patient doesn't quite fit one of the boxes and you } \\
\text { tend to worry... but I think if you can justify whether } \\
\text { actually they've got } 38 \% \text { chance of colorectal cancer } \\
\text { on this (tool) then I don't think they would argue with } \\
\text { that' }\end{array}$ & ${ }_{4}^{\text {Male GP, eRAT }}$ \\
\hline $\begin{array}{l}\text { One size fits all approach } \\
\text { to training }\end{array}$ & $\begin{array}{l}\text { 'Finally, data certainly highlighted that GPs might } \\
\text { decide to refer on the basis of a holistic approach } \\
\text { and, as many respondents emphasized, the approach } \\
\text { of the individual GP and his/her level of clinical } \\
\text { experience also plays a crucial part in the decision } \\
\text { making process' }\end{array}$ & $\begin{array}{l}\text { Author analysis, } \\
\text { eRAT }^{1617}\end{array}$ \\
\hline & $\begin{array}{l}\text { 'Although the tool itself doesn't look that bad on the } \\
\text { training, in terms of the implementation and making } \\
\text { it work in every single practice, I feel that the training } \\
\text { was not bespoke' }\end{array}$ & Male GP, eRAT ${ }^{4}$ \\
\hline
\end{tabular}

\section{Cognitive}

Participation

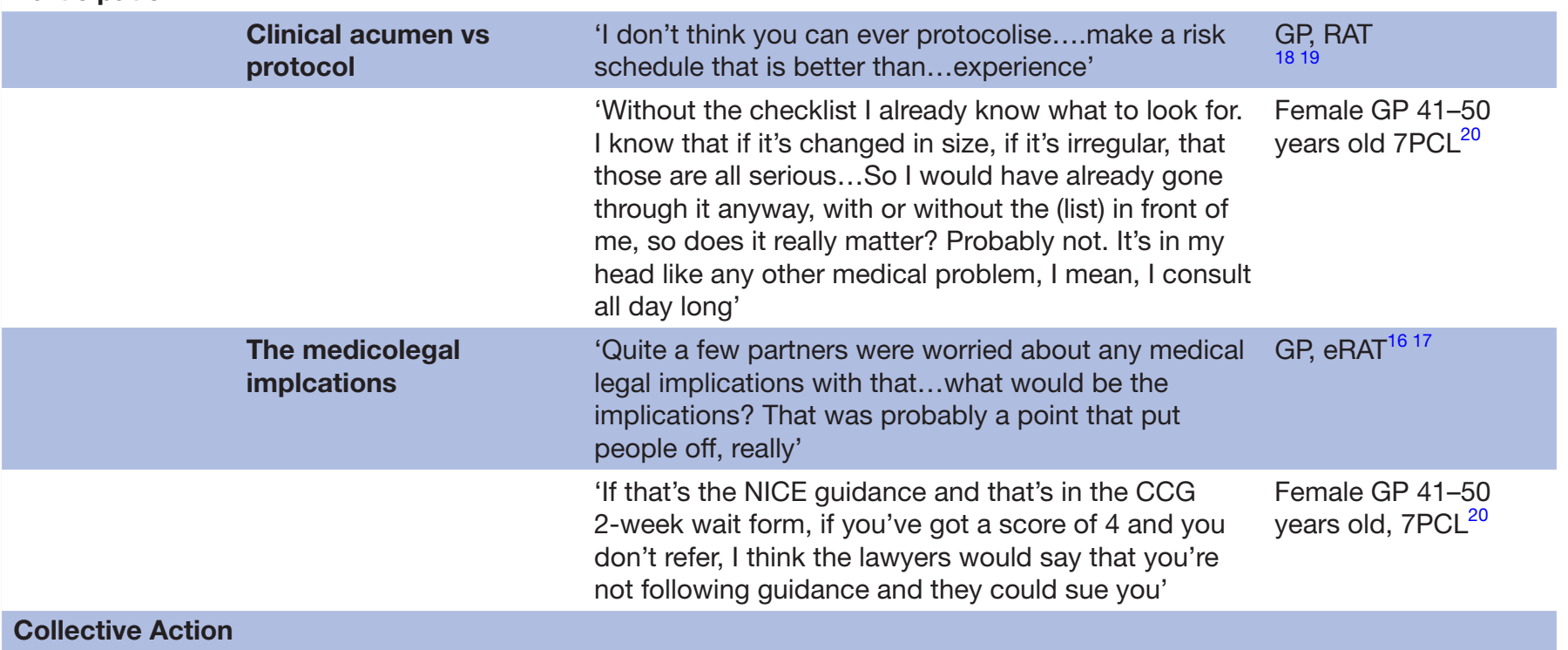

Continued 
Table 4 Continued

\begin{tabular}{|c|c|c|c|}
\hline Domain & Theme & Quote & Source \\
\hline & Increasing awareness & $\begin{array}{l}\text { 'Normally l'd get a few investigations, get the results } \\
\text { back and then based on that say do we need to do } \\
\text { something, or I refer this on based on that. But I guess } \\
\text { if I have a calculator saying it's higher risk, it might } \\
\text { prompt me to make a referral to a specialist a bit } \\
\text { earlier' }\end{array}$ & $\begin{array}{l}\text { Female GP } 31 \text { years } \\
\text { old, QCancer } \\
8\end{array}$ \\
\hline
\end{tabular}

\section{'It probably made us more aware than NICE}

Male GP, eRAT ${ }^{4}$

guidance....it's probably made me more aware of

symptoms which I may have not been as aware of in the past'

Prompt fatigue

Impact of IT integration

Impact of IT integration

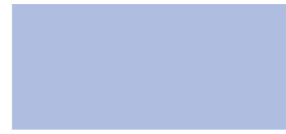

Time as a resource

'I thought it was going to be time consuming using GP, QCancer ${ }^{15}$
the tool. But...that will only be the case in the short
term...it will be time saving in the long term, as the
consultation, the assessments, investigations and
referral processes will be faster'

\section{Reflexive}

Monitoring 'we have all sorts of prompts coming at ...it gets a little Male GP, QCancer bit distracting ...you're trying to sort out and you've got all these messages flashing up at you'

'I suppose the prompt of a photo to be added would be helpful if they need to look through it'

'There was a problem of accessing the tools as they are not integrated in our IT system. It was not easy downloading or googling the tools during patient consultation'

'so much hassle... we had to spend so much time... trying to install it in every single desktop... couldn't do it. I just gave up'

'if it's actually going to make life easier... is it going to improve care for the patient? Or is it ...time really spent in filling up proformas?'

'I thought it was going to be time consuming using consultation, the assessments, investigations and referral processes will be faster'
Male GP 40 years old, 7PCL 20

GP, QCacner ${ }^{15}$

Male GP, eRAT ${ }^{4}$

GP, RAT

1819

GP, QCancer ${ }^{15}$ 'there is a potential for using the tools for screening.... GP, QCancer ${ }^{15}$
They could also be modified for asymptomatic
patients'

'Your chest X-ray is perfectly normal. Your cough settles...I still have to try and convince you to stop smoking, to exercise, to lose weight...it should be used as a relationship tool'
Female GP 50 years old, QCancer ${ }^{8}$
Investigation and referral
patterns

"we were thinking that using the tools in consultation could result in unnecessary...over-referrals...I don't think there will be over-referrals'

'I think our referral thresholds for lower GI have definitely gone down'

Think Cancer

'Yes, I must admit ovarian didn't come so high up... This really said hey, consider ovarian as well"

'If I had a patient with a vague set of symptoms then finding and using the tool showed that it was an amber...I might have followed up the patient in a different way...l'd like to see you again, just to see how these symptoms are, um, rather than leaving it to the patient to contact us"

$\underset{15}{\text { GP, QCancer }}$ Cancer Lead GP, RAT ${ }^{18}$ 19

Male GP 46 QCancer ${ }^{8}$ Cancer Lead GP, RAT ${ }^{18}$ 19

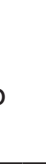

\footnotetext{
eRAT, electronic Risk Assessment Tool; GP, general practitioner; NICE, National Institute for Health and Care Excellence; 7PCL, 7-point checklist; RAT, Risk Assessment Tools.
} 
consultation, time required to train users and the additional effort to continue using the CCDT. Time is at a premium in general practice in the UK: the pressures of the 10 min appointment, ${ }^{24}$ to keep up to date and to attend training.

Participants reported that adjusting to new work practices and integrating new techniques could take additional time, attention and commitment. However, there was evidence of recognition that initial investment may deliver improved patient care. ${ }^{15}$

\section{Reflexive monitoring: GPs reflections on using a new technique}

Reflexive monitoring is the appraisal work that is carried out by individuals utilising the CCDT. ${ }^{18}$

\section{Unintended consequences}

Despite not being an aim of CCDTs, it was recognised that they could be valuable to identify at risk patients or those suitable for screening. ${ }^{8151819}$ In terms of the NPT framework, this highlights how the use of a complex intervention is adapted, based on every day clinical practice and experience.

Another recognised unintended benefit of using the CCDT was in identifying patients' modifiable lifestyle factors. ${ }^{8}{ }^{15}$ This information was used in consultations to try and encourage behavioural change in relation to smoking, weight or alcohol consumption to reduce cancer risk and for general health promotion.

\section{Investigation and referral patterns}

It was acknowledged that the CCDT could reduce overinvestigation and over-referral to secondary care. ${ }^{415-20}$ Conversely, others raised concerns that CCDT would increase the referral rates, but accepted that this could be auditable. ${ }^{151819}$ A reduction in referrals to secondary care would have a positive impact on stretched secondary care services.

\section{'Think cancer'}

All studies demonstrated that CCDTs prompted GPs to 'think cancer, ${ }^{815-20}$ and heightened awareness of relevant signs and symptoms. CCDTs improved the speed of diagnosis and prompted investigations, referrals and counselling of patients. The data revealed that some GPs felt the CCDT elicited earlier review for patients with vague symptoms possibly being caused by an underlying cancer. ${ }^{18} 19$

\section{DISCUSSION}

This qualitative synthesis suggest that GPs recognise the need for awareness of the signs and symptoms of undiagnosed cancer and that the use of CDDTs can help to prompt early referral and diagnosis. As well as these intended benefits, GPs appreciate that CCDTs can be used in ways that benefit the patient doctor interaction. GPs used CCDTs to aid communication with patients about risks of cancer from suspicious as well as vague signs and symptoms and non-suspicious, to reassure anxious patients and to justify legitimate concerns. Using CCDTs in the context of worrying signs and symptoms provides GPs the opportunity to discuss preventative lifestyle changes like weight loss and cessation of smoking which can reduce future risk of developing a cancer.

IT integration issues, interruptions, training and prompts were important factors in how the CCDT functioned in practice and crucial to how participants felt about committing to, and using, the CCDT within consultations.

For CCDTs to be implemented in routine practice, it is important they are thoughtfully developed with collaboration and endorsement from secondary care to ensure compatibility with existing referral criteria. GPs need to be comfortable that the CCDT is another tool in their armamentarium and not seen as a replacement for their gut instinct, experience or factors related to relationships built up over time with patients.

In the field of CCDTs the understanding of risk and its communication needs to be tailored to GPs and patients in a simple, understandable way, ${ }^{25}{ }^{26}$ particularly given the emotive and sensitive nature of suspected cancer and the emphasis on early diagnosis. The discussion of risk with patients and the emphasis on shared decision making (putting patients at the centre of any decisions 'no decision about me without me') ${ }^{27}$ within GP consultations is a key component of universal personalised care. ${ }^{2}$

CCDTs need to be considered clinically valuable, as easy as possible to use and integrate with existing pathways and practices, ${ }^{28}$ while causing minimal disruption to the consultation. Alert fatigue is a recognised consequence of prompts that are generated from the electronic patient record systems. ${ }^{29}$ Clinicians find that electronic prompts interrupt the flow of a consultation, are annoying and are often dismissed without full attention to their contents. This means reminders and alerts that could have a critical clinical impact on a patient are ignored. This is a real concern when the reason is to alert a clinician to an undiagnosed cancer.

GPs acknowledged that any CCDT, and the evidence behind them, need to be acceptable to specialists to whom they refer. GP compliance with guidelines is variable ${ }^{30}$ so discordance between a CCDT and local or national pathways needs to be avoided. Endorsement of CCDT by secondary care would reassure GPs that decision making about referrals was acceptable to both groups. In this context, closer cross-organisational collaboration and trust between healthcare professionals in primary and secondary care could be key to the successful implementation of CCDT. ${ }^{31}$ The Cochrane Review of Interprofessional Collaboration ${ }^{32}$ concludes that to improve professional practice and healthcare outcomes, collaborative work is an area which deserves and is receiving increased academic interest.

The struggle between gut instinct (experience and knowledge of both the medicine and the patient with whom the doctor has a long term therapeutic relationship) 
and protocol is a recognised phenomenon which taps into the GPs' professionalism (autonomy, accountability and responsibility). ${ }^{33}$ No new system should undermine or overrule clinical intuition but should accept that medicine is an art, particularly in the emotively charged arena of cancer diagnosis. Mandating adherence to a particular set of protocols is fraught with barriers. ${ }^{34} \mathrm{~A}$ recent systematic review and meta-analysis found that the GP's 'gut feeling' was an important predictor of a cancer diagnosis. ${ }^{35}$

This is the first theoretically informed qualitative synthesis of the views and experiences of general practitioners in relation to the implementation of Clinical Cancer Decision Tools (CCDTs) used within the rimary are consultation. The systematic and theory informed approach to synthesis allowed the identification of some generic and transferable issues relevant across a range of different CCDTs and contexts.

The small number of studies and data available for inclusion in this review limited detailed comparative analysis between CCDTs and contexts, and further work in this area may be beneficial. The quality and depth of the findings are limited by the quality of the studies included, and any bias of the original authors. Unfortunately the he small number of studies and data available for inclusion in this review limited the ability to carry out a more detailed comparative analysis between CCDTs and contexts, and further work in this area may be beneficial.

The studies are recent (2011 onwards) reflecting modern primary care practice but predominantly within the English NHS. The CCDTs evaluated are different: in format, in how the individual patient's risk of cancer is calculated and their affinity with existing referral criteria. Nonetheless, this systematic and theory informed approach to synthesis identified some generic and transferable issues relevant across different CCDTs.

The ubiquitous electronic clinical decision aid takes many forms in primary care and this study reflects GPs' experience from just those designed to improve recognition of signs and symptoms that could be caused by an undiagnosed cancer. There are lessons to be learnt from the experience of successful implementation of electronic decision aids in other clinical domains such as cardiovascular disease. ${ }^{36}$

More evidence is needed that suspected cancer referrals generated from the use of CCDTs lead to identification of cancers at an earlier stage with subsequent impact on treatment outcomes. This evidence would further legitimise the use of CCDTs along with endorsement of their use by national guidance bodies for cancer pathways such as NICE in England.

\section{CONCLUSIONS}

Comprehensive cooperative working between primary and secondary care in planning, designing and implementing CCDTs will benefit clinicians, patients, quality of healthcare and take account of scarce resources.
Stakeholder consultation and involvement should be regarded as essential aspects of healthcare innovation and implementation.

The value that clinicians place on their clinical acumen and their desire for this to be recognised makes them wary to rely solely on protocol driven decision making. There will always be clinicians who find decision aids reassuring, those who find them helpful as an aide-memoire and those that find them a nuisance and will not use them at all. An ideal CCDT is one that does not undermine clinical instincts but supports and enhances them.

CCDTs can be a helpful adjunct to clinical work in primary care, but without careful development legitimising their use as well as consideration of training and IT integration with secondary care and IT systems, they may remain to be perceived as superfluous to clinical acumen and experience. Stakeholder consultation and involvement should be regarded as essential aspects of healthcare innovation and implementation.

This theoretically informed synthesis of existing qualitative work has helped to identify key themes and issues that influence the use and implementation of CCDTs across cancers, tools and settings. These insights can help to inform future development and implementation of CCDTs and fuller integration within policy and referral guidelines.

Twitter Paula Theresa Bradley @drptbradley and Richard D Neal @richarddneal

Contributors PTB: protocol, literature search, data selection, data analysis, article. NH: protocol review, data selection, data analysis, manuscript editing. GM: data analysis, manuscript editing. RDN, VP and SW: supervision at all stages

Funding PTB is a PhD candidate and Extended Integrated Training Programme GP Trainee in a post funded by Health Education England North East as part of the Northumbria General Practice Training Programme.

Competing interests None declared.

Patient consent for publication Not required.

Provenance and peer review Not commissioned; externally peer reviewed. Data availability statement There are no data in this work.

Supplemental material This content has been supplied by the author(s). It has not been vetted by BMJ Publishing Group Limited (BMJ) and may not have been peer-reviewed. Any opinions or recommendations discussed are solely those of the author(s) and are not endorsed by BMJ. BMJ disclaims all liability and responsibility arising from any reliance placed on the content. Where the content includes any translated material, BMJ does not warrant the accuracy and reliability of the translations (including but not limited to local regulations, clinical guidelines, terminology, drug names and drug dosages), and is not responsible for any error and/or omissions arising from translation and adaptation or otherwise.

Open access This is an open access article distributed in accordance with the Creative Commons Attribution Non Commercial (CC BY-NC 4.0) license, which permits others to distribute, remix, adapt, build upon this work non-commercially, and license their derivative works on different terms, provided the original work is properly cited, appropriate credit is given, any changes made indicated, and the use is non-commercial. See: http://creativecommons.org/licenses/by-nc/4.0/.

ORCID iDs

Paula Theresa Bradley http://orcid.org/0000-0002-1908-1053

Richard D Neal http://orcid.org/0000-0002-3544-2744 


\section{REFERENCES}

1 Abdel-Rahman M, Stockton D, Rachet B, et al. What if cancer survival in Britain were the same as in Europe: how many deaths are avoidable? Br J Cancer 2009;101 Suppl 2:S115-24.

2 NHS. Nhs long term plan, 2019. Available: https://www.longtermplan. nhs.uk/wp-content/uploads/2019/01/nhs-long-term-plan-june-2019. pdf

3 England N. QOF quality improvement domain 2020/21 - early diagnosis of cancer, 2020. Available: https://www.england.nhs.uk/ wp-content/uploads/2020/02/20-21-qof-qi-cancer.pdf [Accessed 13 Dec 2020].

4 Moffat JI, Green TL. Clinical decision support tool for cancer (CDS) project: evaluation report to the Department of health, 2014 Available: https://www.macmillan.org.uk/ images/cds-executivesummary_tcm9-291978.pdf [Accessed 25 Feb 2020].

5 Walter FM, Morris HC, Humphrys E, et al. Effect of adding a diagnostic aid to best practice to manage suspicious pigmented lesions in primary care: randomised controlled trial. BMJ 2012;345:e4110.

6 Walter FM, Prevost AT, Vasconcelos J, et al. Using the 7-point checklist as a diagnostic aid for pigmented skin lesions in general practice: a diagnostic validation study. Br J Gen Pract 2013;63:e345-53.

7 Price S, Spencer A, Medina-Lara A, et al. Availability and use of cancer decision-support tools: a cross-sectional survey of UK primary care. Br J Gen Pract 2019;69:e437-43.

8 Chiang PP-C, Glance D, Walker J, et al. Implementing a QCancer risk tool into general practice consultations: an exploratory study using simulated consultations with Australian general practitioners. $\mathrm{Br} \mathrm{J}$ Cancer 2015;112 Suppl 1:S77-83.

9 Chima S, Reece JC, Milley K, et al. Decision support tools to improve cancer diagnostic decision making in primary care: a systematic review. Br J Gen Pract 2019;69:e809-18.

10 et alMay C, Rapley T, Mair FS. Normalization Process Theory On-line Users' Manual, Toolkit and NoMAD instrument, 2015. Available: http://www.normalizationprocess.org [Accessed 18/06/2019].

11 Booth A, Brice A. Evidence-based practice for information professionals : a handbook. London: Facet, 2004.

12 Critical Appraisal Skills Programme. Casp (qualitative checklist), 2019. Available: https://casp-uk.net/casp-tools-checklists/ [Accessed 31 Oct 2019].

13 Garside R. Should we appraise the quality of qualitative research reports for systematic reviews, and if so, how? Innovation: The European Journal of Social Science Research 2014;27:67-79.

14 Brunton G, Oliver S, Thomas J. Innovations in framework synthesis as a systematic review method. Res Synth Methods 2020;11:316-30.

15 Akanuwe J. Exploring service user and practitioner perspectives of using cancer risk assessment tools in primary care consultations. University of Lincoln, 2018.

16 Dikomatis TG, Macleod, U T. MacMillan electronic risk assessment tool summary: dealing with uncertainty: a qualitative evaluation of the usability and acceptability of an electronic risk assessment tool to aid cancer diagnosis in general practice: supportive care, early diagnosis and advanced disease (SEDA) Research Group. centre for health and population sciences (CHAPS), 2012. Available: https:// www.macmillan.org.uk/documents/aboutus/health_professionals/ earlydiagnosis/summaryeratsreport(oct2012).pdf

17 Dikomitis L, Green T, Macleod U. Embedding electronic decision-support tools for suspected cancer in primary care: a qualitative study of GPs' experiences. Prim Health Care Res Dev 2015;16:548-55

18 Green T, Martins T, Hamilton W, et al. Exploring GPs' experiences of using diagnostic tools for cancer: a qualitative study in primary care. Fam Pract 2015;32:101-5.

19 Hamilton W, Green T, Martins T, et al. Evaluation of risk assessment tools for suspected cancer in general practice: a cohort study. $\mathrm{Br} J$ Gen Pract 2013;63:e30-6.

20 Pannebakker MM, Mills K, Johnson M, et al. Understanding implementation and usefulness of electronic clinical decision support (eCDS) for melanoma in English primary care: a qualitative investigation. BJGP Open 2019;3:bjgpopen18X101635.

21 National Institute of Care and Health Excellence. Melanoma and pigmented lesions, 2017. Available: https://cks.nice.org.uk/topics/ melanoma-pigmented-lesions/diagnosis/assessment/ [Accessed 20 Dec 2020].

22 Berger R. Now I see it, now I don't: researcher's position and reflexivity in qualitative research. Qualitative Research 2015;15:219-34.

23 Murray E, Treweek S, Pope C, et al. Normalisation process theory: a framework for developing, evaluating and implementing complex interventions. BMC Med 2010;8:63.

24 Hobbs FDR, Bankhead C, Mukhtar T, et al. Clinical workload in UK primary care: a retrospective analysis of 100 million consultations in England, 2007-14. Lancet 2016;387:2323-30.

25 Ahmed $\mathrm{H}$, Naik $\mathrm{G}$, Willoughby $\mathrm{H}$, et al. Communicating risk. BMJ 2012;344:e3996.

26 Naik G, Ahmed H, Edwards AGK. Communicating risk to patients and the public. Br J Gen Pract 2012;62:213-6.

27 Coulter A, Collins A. Making shared decision-making a reality : no decision about me, without me. London: King's Fund, 2011.

28 Maniatopoulos G, Procter R, Llewellyn S, et al. Moving beyond local practice: reconfiguring the adoption of a breast cancer diagnostic technology. Soc Sci Med 2015;131:98-106.

29 Backman R, Bayliss S, Moore D, et al. Clinical reminder alert fatigue in healthcare: a systematic literature review protocol using qualitative evidence. Syst Rev 2017;6:255.

30 Lugtenberg M, Burgers JS, Besters CF, et al. Perceived barriers to guideline adherence: a survey among general practitioners. BMC Fam Pract 2011;12:98.

31 Maniatopoulos G, Haining S, Allen J, et al. Negotiating commissioning pathways for the successful implementation of innovative health technology in primary care. BMC Health Serv Res 2019;19:648.

32 Reeves S, Pelone F, Harrison R, et al. Interprofessional collaboration to improve professional practice and healthcare outcomes. Cochrane Database Syst Rev 2017;6:CD000072.

33 Kristensen N, Nymann C, Konradsen H. Implementing research results in clinical practice- the experiences of healthcare professionals. BMC Health Serv Res 2016;16:48.

34 O'Leary P, Carroll N, Richardson I. The Practitioner's Perspective on Clinical Pathway Support Systems. 2014 leee International Conference on Healthcare Informatics, 2014:194-201.

35 Smith CF, Drew S, Ziebland S, et al. Understanding the role of GPs gut feelings in diagnosing cancer in primary care: a systematic review and meta-analysis of existing evidence. $\mathrm{Br} \mathrm{J}$ Gen Pract 2020;70:e612-21.

36 Ban J-W, Perera R, Stevens R. Gps' familiarity with and use of cardiovascular clinical prediction rules: a UK survey study. BJGP Open 2020;4:bjgpopen20X101081. 